\title{
Hospital Discharge Strategy at a Governmental Hospital in Qatar
}

\author{
Dr. Almunzer Zakaria*1, Dr. Sara Kazkaz ${ }^{2}$ and Dr. Moza Alishaq ${ }^{3}$ \\ 1,2Quality Improvement and Patient Safety Department, Hamad medical corporation, Qatar \\ ${ }^{3}$ Corporate Quality Improvement and Patient Safety Department, Hamad medical Corporation, Qatar
}

*Corresponding author: Dr. Almunzer Zakaria, DBA/Healthcare Management, Quality Improvement and Patient Safety Department, Alwakra Hospital, Hamad medical corporation, Qatar

Received Date: June 10, 2020

Published Date: July 02, 2020

\begin{abstract}
Preparing a patient for hospital discharge takes time, especially when complicated by medication preparation and reconciliation, patient education, and discharge summary documentation. Therefore, the healthcare managers and administrators should have tangible policies for discharging patients to avoid unnecessary readmissions linked to wasteful hospital expenditures. A single case study design was applied to investigate the varied inputs from different administrators within one setting and obtained information related to optimal patient discharge practices bounded by time and place. The hospital had six departments, the departments including pediatrics, obstetrics and gynecology, surgery, burns, medicine, and an intensive care unit. One toolkit designed to reduce readmission rates is the Re-Engineered Discharge (RED) toolkit. This strategy is an evidenced-based approach with the three-fold aim of ensuring that patients understand how to care for themselves, reducing emergency visits and readmissions, and improving patient satisfaction. As for implementing RED, several specific indicators could be used depending on the context specific to the hospital. These indicators should be meaningful, credible, feasible, and timely. As a result of this study, both patient educations, planning for outpatient appointments and telephone follow-up by clerical or nursing staff were shown to have successfully reduced readmission rates for patients discharged to home. The hospital setting involved in the study introduced the admission-discharge lounge that helps, first, to accommodate patients that are already discharged in the inpatient but are not yet ready to leave the hospital for personal reasons. This prompts the process of clearing bed spaces for other admissions. Second, is by transferring the patient to the admission-discharge lounge, giving the patient and families time to support the discharge instruction education because now patients receive the instructions twice, once from the inpatient, and also from the lounge.
\end{abstract}

Keywords: Hospital Discharge; RED Toolkit; Readmissions; admission-discharge lounge

Abbreviations: RED toolkit: Re-Engineered Discharged toolkit, AHRQ: the Agency for Health Research and Quality

\section{Introduction}

Preparing a patient for hospital discharge takes time, especially when complicated by medication preparation and reconciliation, patient education, and discharge summary documentation [13]. Discharge preparation differs for each patient due to unique admitting history, a physical examination, and detailed medical education. A good hospital discharge transition is as vital as a thorough admission evaluation [4]. Reasons for readmissions may vary, but may include a lack of information at discharge from hospital and a clinical preventive action, such as preparing the patients with proper care planning while at the hospital [5]
.Therefore, the healthcare managers and administrators should have tangible policies for discharging patients to avoid unnecessary readmissions linked to wasteful hospital expenditures [6].

\section{Materials and Methods}

A qualitative method was used to explore phenomena in which limited previous understanding existed; thus, the findings may produce new insights and practices. A single case study design was applied to investigate the varied inputs from different administrators within one setting and obtained information related to optimal patient discharge practices bounded by time and 
place. A variety of data collection techniques were used, including an interview protocol, semi-structured interviews, document review, and data analysis. Methodological triangulation helped to strengthen the research and gain data from the facility's Health Information Management Office. The data analysis process included coding and clustering the interview data and identifying common trends in the findings; and generating themes that aligned with the central research question and conceptual framework.

The documents reviewed were monthly data on the percentage of discharges from January 2018 to April 2019. Documentation supplemented the qualitative interviews and helped to provide a deeper understanding of the scope of the phenomenon. The hospital had a capacity of 326 beds and an average of 50 discharges and 65 admissions per day, in Qatar. The hospital had six departments, the departments including pediatrics, obstetrics and gynecology, surgery, burns, medicine, and an intensive care unit. which provided the setting for this study. Understanding that procedures and patient outcomes varied between departments, all six departments provided significant inputs to conduct the investigation. Interviews were conducted with four hospital administrators and included reviews of relevant documentation offered by the administrators [7]. The administrators were asked about strategies that were used upon discharge to assist the patient to continue the recovery process to avoid readmissions and learn about the different services available in the hospital to support patients post-discharge.

\section{Results and Discussion}

Efforts to improve how the discharge plan is prepared were notable. Aside from interviews, data from the Health Information Management Office indicated that the discharge rates, from January 2018 to March 2019, were fluctuating. The trends of discharge depend on the specific units in the hospitals. Dramatic drops occurred in the obstetrics/gynecology unit and burn units. The pediatric unit, surgical unit, medical unit, and intensive care unit showed almost similar trends in discharge rates; however, there was no dramatic drop or increase noticeable. All units' performance showed that the discharge rate for March 2018 was similar to the baseline taken in January 2018.

One toolkit designed to reduce readmission rates is the ReEngineered Discharge (RED) toolkit. This strategy is an evidencedbased approach with the three-fold aim of ensuring that patients understand how to care for themselves, reducing emergency visits and readmissions, and improving patient satisfaction. To structure and fully operationalize the RED into the patient care system, may take at least six months. The RED was prepared by the Boston University Medical Center, in cooperation with the Agency for Health Research and Quality (AHRQ). The RED has 12 components, which are

a) ascertain the need for and obtain language assistance,

b) make appointments for follow-up care, c) plan for the follow-up of results from tests or labs that are pending at discharge,

d) organize post-discharge outpatient services and medical equipment,

e) identify the correct medicines and a plan for the patient to obtain them,

f) reconcile the discharge plan with national guidelines,

g) teach a written discharge plan the patient can understand,

h) Educate the patient about his/her diagnosis and medicines,

i) review with the patient what to do if a problem arises,

j) assess the degree of the patient's understanding of the discharge plan,

k) expedite transmission of the discharge summary to clinicians accepting care of the patient, and

l) provide telephone reinforcement of the discharge plan [8].

As for implementing RED, several specific indicators could be used depending on the context specific to the hospital. These indicators should be meaningful, credible, feasible, and timely. Furthermore, Implementation should measure whether RED was delivered to target patients, whether the correct information was collected, whether evidence-based care was delivered, whether appropriate follow-up care was arranged, whether patients were prepared for discharge, and whether patients received postdischarge care [8].

Once the patient returns home following a hospitalization, the RED captures key junctions in which the discharge plan failed; for instance, when the discharge education was not properly given or if the patient or family did not understand what the patient needed to do once home. If this occurs, patients will most likely not follow the medication schedules which in turn will make their recovery slower, sometimes making their condition worse than when patients were discharged from the hospital, forcing them to return to the emergency department within a few days. Poor patient education can also lead to problems in medication instructions.

Sometimes, incomplete patient education is given to the patient before the patient was discharged, and that includes improper medicine reconciliation. Alternatively, the education was complete, but the patient didn't understand the instructions completely because of the language barrier. As a result of this study, the hospital introduced the admission-discharge lounge that helps nursing staff to clear bed spaces for the pending admissions in the emergency departments and reinforces the discharge instruction education to the patients and families. The nursing staffs are encouraged to make sure that the discharge plan is completed 24 hours prior to 
discharge. Early preparation gives time for the patients to fully understand the education provided and gives the patient time to think of possible questions to ask the staff regarding parts of the plan that patients and families didn't understand.

\section{Conclusion}

As a result of this study, both patient education, planning for outpatient appointments and telephone follow-up by clerical or nursing staff were shown to have successfully reduced readmission rates for patients discharged to home [9]. The RED program was introduced to streamline the patient discharge process and to decrease the readmissions at hospitals. The RED program is comprised of 12 components which included discharge planning and patient teaching [10]. Client teaching, for instance, included teaching patients a written discharge plan in terms that client. Education related to the clients' diagnosis and medicines must be provided at discharge to ensure the client is well-prepared to continue a successful healing process at home. The discharge plan must include telephone reinforcement, expediting the transmission of the discharge summary to patients. In summarizing the effectiveness of RED, there was a wide variability in the fidelity of the interventions, engaged leadership and multidisciplinary implementation teams as keys to success, and some challenges such as timely follow-up appointments, transmitting discharge summaries to outpatient clinicians, and leveraging information technology. The RED showed improvement in 30-day readmission rates.

As mentioned, the hospital setting involved in the study introduced the admission-discharge lounge that helps, first, to accommodate patients that are already discharged in the inpatient but are not yet ready to leave the hospital for personal reasons. This prompts the process of clearing bed spaces for other admissions. Second, is by transferring the patient to the admissiondischarge lounge, giving the patient and families time to support the discharge instruction education because now patients receive the instructions twice, once from the inpatient, and also from the lounge. If patients and families did not understand any instructions during the first time, both have another chance to gain clarity, or ask further questions. The final key advantage is the availability and set up of home care.

Moreover, the discharge plan is completed 24 hours prior to discharge. By doing so, medical staff are relieved from the pressure and time to finish the plan on the day of discharge. This arrangement helps the patient to perceive the discharge instructions and gives time to clarify any point in the plan that the patient and families didn't understand. Hospitals require more protocols and guidelines for the effective treatment and release of the patient. If hospital staffs effectively treat the patient, the patient will not return, and therefore, hospitals will have more empty beds for other patients which will have a positive impact on pending patients in the emergency department.

\section{Acknowledgement}

None.

\section{Conflict of Interest}

No conflict of interest.

\section{References}

1. Jenq G Y, Doyle M M, Belton B M, Herrin J, Horwitz L I (2016) Quasiexperimental evaluation of the effectiveness of a large-scale readmission reduction program. Journal of the American Medical Association Internal Medicine 176: 681-690.

2. Wasfy J H, Zigler C W, Choirat C, Wang Y, Dominici F, Yeh R W (2017) Readmission rates after passage of the hospital readmissions reduction program: a pre-post analysis. Annals of Internal Medicine166: 324-331.

3. Zuckerman R B, Sheingold S H, Orav E J, Ruhter J, Epstein A M (2016) Readmissions, observation, and the hospital readmissions reduction program. New England Journal of Medicine 374: 1543-51.

4. Kripalani S (2007) Care Transitions.

5. Hallgren J, Aslan AKD (2018) Risk factors for hospital readmission among Swedish older adults. Eur Geriatr Med 9(5): 603-611.

6. Upadhyay S, Stephenson A L, Smith D G (2019) Readmission rates and their impact on hospital financial performance: a study of Washington hospitals. The Journal of Health Care Organization, Provision, and Financing, pp. 56.

7. Zakaria A, Faint CA, Kazkaz S (2020) Readmission rate reduction strategies in general hospital setting. Aging Male. 22: 1-4.

8. Jack B, Paasche-Orlow M, Mitchell S, Forsythe S, Martin J, et al. (2013) Re-Engineered Discharge (RED) Toolkit.

9. Kripalani S, Theobald C N, Anctil B, Vasilevskis, E E (2014) Reducing hospital readmission rates: current strategies and future directions. Annual Review of Medicine 65: 471-485.

10. Mitchell S E, Martin J, Holmes S, van Deusen Lukas C, Cancino, R, et al. (2016) How hospitals reengineer their discharge processes to reduce readmissions. Journal for Healthcare Quality: Official Publication of the National Association for Healthcare Quality 38(2): 116. 